\title{
Optical transmission subsystems and techniques special issue based on selected IEEE ICOCN 2014 papers
}

\author{
Changyuan Yu' ${ }^{1}$ - Zhongqi Pan ${ }^{2} \cdot$ Qi Yang ${ }^{3}$ - Gangxiang Shen ${ }^{4}$
}

Published online: 1 October 2015

(C) Springer Science+Business Media New York 2015

The 13th International Conference on Optical Communications and Networks-IEEE ICOCN 2014 (http:// http://www. ontrc.org/icocn/) was held in Suzhou on November 9-10, 2014.

IEEE ICOCN aims to provide a premier opportunity for professionals, experts, engineers, scientists, and industrial people worldwide in the field of research, development, and applications of photonics to share and exchange their experience. IEEE ICOCN 2014 included three technical tracks: (1) optical networks, (2) optical transmission subsystems and techniques, and (3) photonic devices and integration.

IEEE ICOCN 2014 was a two-day event organized by Optical Network Technology Research Center (ONTRC) in School of Electronic and Information Engineering, Soochow University. IEEE ICOCN 2014 was technically sponsored by IEEE Photonics Society. The conference was also supported by National Natural Science Foundation of China

$\triangle$ Changyuan Yu

eleyc@nus.edu.sg

Zhongqi Pan

zpan@louisiana.edu

Qi Yang

qyang@wri.com.cn

Gangxiang Shen

shengx@ suda.edu.cn

1 National University of Singapore, Singapore 117583, Singapore

2 University of Louisiana at Lafayette, Lafayette, LA 70504, USA

3 State Key Laboratory of Optical Communication Technologies and Networks, Wuhan 430074, People's Republic of China

4 Soochow University, Suzhou 215006, People's Republic of China
(NSFC), Zhongtian Broadband Technology, Electronic Institute of Suzhou, and the City Government of Suzhou.

We accepted more than 120 papers by the authors from more than 13 countries and regions, among which 59 are invited talks. All the papers were carefully reviewed by more than 30 Technical Program Committee (TPC) members and external reviewers, with each paper evaluated by at least two reviewers. All these papers and talks are organized into eight lecture-style oral sessions and one interactive poster session.

Based on the comments received from the reviewers, the authors of a limited number of accepted papers in the area of optical transmission subsystems and techniques were invited to submit an extended version of their work for possible publication in this Optical Transmission Subsystems and Techniques Special Issue of the Springer Photonic Network Communications (PNET) journal, which again went through a peer review process. Ten papers among them were selected for publication. A brief summary on these papers is provided next.

In "Power-efficient schemes using directly modulated lasers for intensity modulation and direct detection OFDMPONs," X. Gong, L. Guo, and Y. Liu propose an improved Hughes-Hartogs Algorithm (IHHA) to adjust the number of bits and power level allocated for each subcarrier, with an objective to minimize the electrical transmitting power of all subcarriers. The OFDM signal is generated according to the bit/power allocation result of IHHA. Simulation results show that the system has high power efficiency by using IHHA, and bit error rate (BER) satisfies the forward error correction (FEC) limitation after $100 \mathrm{~km}$ single-mode-fiber (SMF) transmission.

In "Performance of ADC resolution limited optical coherent receivers with DA-ML carrier phase estimation," Y. Fan, J. Chen, Y. Zhang, and C. Yu investigate the performance of decision-aided maximum-likelihood (DA-ML) carrier phase 
estimation (CPE) algorithm in analog-to-digital converter (ADC) resolution-limited optical coherent receivers. Simulation and theoretical analysis both indicate that the optimal ADC quantization range equals to the average amplitude of received symbols which carry the information symbols with the highest energy on the constellation.

In "DFT-based optical offset-QAM OFDM: analytical, numerical, and experimental studies," J. Zhao investigates discrete Fourier transform-based offset quadrature amplitude modulation (offset-QAM) orthogonal frequency division multiplexing (OFDM) technology. It is found that channel estimation in offset-QAM OFDM is different from that in conventional OFDM (C-OFDM) due to the residual cross talk terms and requires particular study. Numerical and experimental results show that DFT-based offset-QAM OFDM can greatly enhance the net data rate for fiber transmissions compared to C-OFDM and exhibit lower complexity than Nyquist FDM.

In "Investigation of nonlinear effects in few-mode fibers," Z. Pan, Y. Weng, and J. Wang exploit nonlinear optical effects for future implementations of space-division-multiplexing (SDM) fiber systems. An ultrafast all-optical simultaneous wavelength and mode conversion scheme based on intermodal four-wave mixing (IM-FWM) is proposed, with the capability of switching state of polarization (SOP) and mode degeneracy orientation (MDO). Under this scheme, crosspolarization modulation (XPolM) and cross-mode modulation (XMM) can be achieved.

In "Data-aided linear fitting blind phase estimation method for coherent optical OFDM system," M. Pan, X. Zhang, J. $\mathrm{Li}$, and $\mathrm{Z}$. Li propose a novel data-aided linear fitting-based (DALF-based) blind phase estimation method for coherent optical orthogonal frequency division multiplexing (COOFDM). Simulation results show that the performance of the proposed DALF-based method is better than the conventional one with higher SE and has the potential in the large capacity CO-OFDM systems.

In "Enhanced performance of phase-conjugated OFDM subcarriers using digital coherent superposition," X. Yi, D. Zeng, H. Zhou, C. Tang, and K.Qiu summarize their techniques to improve phase noise tolerance in coherent optical OFDM based on phase-conjugated twin wave (PCTW). The approaches can cancel the intercarrier interference (ICI) due to phase noise. The tolerance to fiber nonlinearity is also enhanced.

In "Passive phase correction for stable radio frequency transfer via optical fiber," S. Pan, J. Wei, and F. Zhang establish an analytical model for the stable RF transfer system based on passive phase correction and develop the techniques in solving the problems of the proposed method. Future prospects and perspectives are also discussed.

In "Design and performance evaluation of narrowband rectangular optical filter based on stimulated Brillouin scat- tering in fiber," L. Yi, W. Wei, M. Shi, Y. Jaouen, and W. Hu propose a rectangular optical filter based on stimulated Brillouin scattering (SBS) in fiber with tunable bandwidth from $50 \mathrm{MHz}$ to $4 \mathrm{GHz}$ at $15-\mathrm{MHz}$ tuning resolution. The steepedged rectangular shape of the filter is precisely controlled utilizing digital feedback compensation of the multitone pump light. The amplification performance with different gains for OFDM signal is validated, which shows the potential capability of the filter in the fields of optical signal processing.

In "Flexible high-order QAM transmitters for elastic optical networks," G. LU, T. Sakamoto, and T. Kawanishi demonstrate two flexible high-order quadrature amplitude modulation (QAM) transmitter schemes. The schemes provide different approaches to configure a flexible high-order QAM transmitter, either using cascaded off-the-shelf modulators, or via highly integrated monolithic modulator. These flexible and reconfigurable transmitter schemes exhibit different characteristics in terms of system performance, which provides the network operators different flexible transmitter solutions suitable for different application scenarios in elastic optical networks.

In "Chip-Scale optical interconnects and optical data processing using silicon photonic devices," J. Wang reviews the recent progress in the design, modeling, and fabrication of silicon photonic devices and their applications in chipscale optical interconnects and optical data processing with advanced modulation formats. The obtained theoretical and experimental results indicate possible integration of optical communication functions on a monolithic chip.

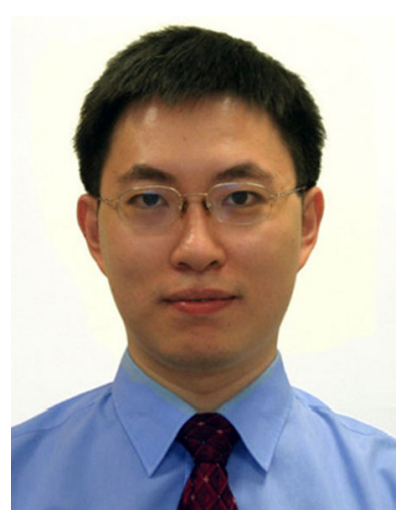

Changyuan Yu joined Department of Electrical and Computer Engineering, National University of Singapore (NUS) in December 2005. He has founded and led the Photonic System Research Group in NUS since then. He has supervised five postdoc research fellows and 15 Ph.D. students (7 graduated) in his group. He is also a $25 \%$ joint senior scientist with A*STAR Institute for Infocomm Research (I2R). He received B.S. in Applied Physics and B. Economics in Management from Tsinghua Univ., China, in 1997, M.S. in Electrical and Computer Engineering from the Univ. of Miami, USA, in 1999, and Ph.D. in Electrical Engineering from the Univ. of Southern California, USA, in 2005. He was a visiting researcher at NEC Labs America in 2005. His research focuses on photonic devices, subsystems, and optical fiber communication and sensor systems. Dr. Yu has authored/co-authored six book chapters and over 240 research papers on the peer-reviewed journals and the prestigious conferences (39 invited, including OFC2012 in USA). He has served in technical program committee or organizing committee for $50+$ international conferences (including TPC member of OFC2014-2016, Chair of SPP- 
Com2015, and TPC chair of SPPCom2014 in USA). His group won six best paper awards in conferences and the championship in biomedical area in the 3rd China Innovation and Entrepreneurship Competition (out of $10,000+$ competitors) in 2014 .

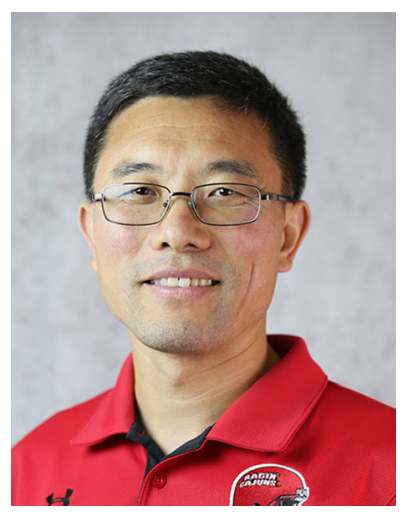

Zhongqi Pan received his Ph.D. degree in Electrical Engineering from the University of Southern California. He has worked as a Lecturer and Assistant Professor at Tsinghua University, and a Medical Image Processing System Engineer at 301 Hospital, Beijing. He joined the University of Louisiana at Lafayette in 2003 and is currently a full professor at the Department of Electrical and Computer Engineering. Dr. Pan's research is in the area of optical fiber communications, including photonic devices, wavelength-division-multiplexing (WDM) technologies, optical performance monitoring, coherent optical communications, and space-division-multiplexing (SDM) technologies. He has authored/co-authored over 130 publications on optical devices, nonlinear optics, and fiber communications systems. He also holds five US patents and 1 China patent. He has received National Invention Award in China in 1998 and the award for Outstanding Academic Advisor in 2006 and Excellence in Academic Advising in 2012 from UL Lafayette. Dr. Pan is an OSA and IEEE senior member. He currently serves as a member of OSA's External Relations Advisory Committee. He also has served as Committee member and the session Chair for SPIE Photonics West, Subcommittee Co-Chair for the International Conference on Optical Communications and Networks (ICOCN), the Technical Program Committee for the IEEE International Conference on Communications Systems, the IEEE Photonics Global Conference, and the IEEE Annual Wireless and Optical Communications Conference (WOCC), and as reviewers for numerous IEEE and OSA conferences/journals.

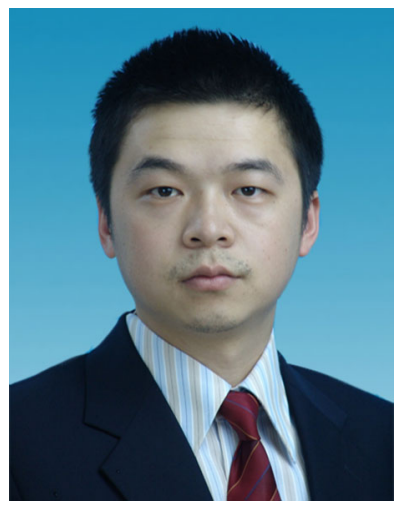

Qi Yang received the B.E. and M.E. degrees in electronic and information engineering from Huazhong University of Science and Technology, Wuhan, China, in 2004 and 2006, and Ph.D. degree in electrical and electronic engineering at the University of Melbourne, Melbourne, Australia, in 2010, respectively. From 2008 to 2009, Dr. Yang worked as intern student with Bell Labs, Alcatel-lucent Technologies, Murray Hill, NJ. Since 2010, we have been with the State Key Laboratory of Optical Communication Technologies and Networks, Wuhan, China. His current research intersects include highspeed coherent transmission. He has published more than 90 journal and conference papers.

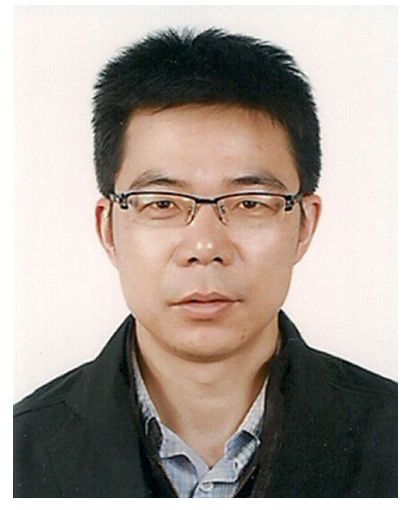

Gangxiang Shen [S'98-M'99 - SM' 12] received his B.Eng. degree from Zhejiang University, China; his M.Sc. degree from Nanyang Technological University, Singapore; and his Ph.D. degree from the University of Alberta, Canada, in January 2006. He is a Distinguished Professor with the School of Electronic and Information Engineering of Soochow University in China. Before he joined Soochow University, he was a Lead Engineer with Ciena, Linthicum, Maryland. He was also an Australian ARC Postdoctoral Fellow with University of Melbourne. His research interests include integrated optical and wireless networks, spectrum-efficient optical networks, and green optical networks. He has authored and co-authored more than 100 peer-reviewed technical papers. He is a Lead Guest Editor of IEEE JSAC Special Issue on "Next-Generation Spectrum-Efficient and Elastic Optical Transport Networks," and a Guest Editor of IEEE JSAC Special Issue on "Energy-Efficiency in Optical Networks." $\mathrm{He}$ is an associated editor of IEEE/OSA JOCN and an editorial board member of Optical Switching and Networking. He is a Secretary for the IEEE Fiber-Wireless (FiWi) Integration Sub-Technical Committee. He received the Young Researcher New Star Scientist Award in the "2010 Scopus Young Researcher Award Scheme" in China. He was a recipient of the Izaak Walton Killam Memorial Award from the University of Alberta and the Canadian NSERC Industrial R\&D Fellowship. 